\title{
CONVERGENCE OF THE MAXIMUM PROBABILITY OF SUCCESS IN THE PROBLEM OF QUANTILE HEDGING FOR A MODEL OF AN ASSET PRICE PROCESS WITH LONG-RANGE DEPENDENCE
} UDC 519.21

\author{
M. V. BRATYK, YU. V. KOZACHENKO, AND YU. S. MISHURA
}

\begin{abstract}
The convergence in probability of the sets of maximum probability of success is studied in the problem of quantile hedging for a model of an asset price process involving Brownian and fractional Brownian motions.
\end{abstract}

\section{INTRODUCTION}

The problem of hedging contingent claims is well studied for complete arbitrage-free financial markets. Suppose an investor wants to ensure that a contingent claim $H$ is hedged at a fixed moment $T>0$ and assume that the investor capital is modeled by a semimartingale $X=\left\{X_{t}, t \geq 0\right\}$. As is known, a necessary and sufficient condition for such a perfect hedging is that the initial capital $H_{0}$ satisfies $H_{0}=E_{P^{*}}(H)$, where $E_{P^{*}}$ denotes the expectation with respect to the unique martingale measure $P^{*}$ (in other words, with respect to the measure such that $X$ is a martingale with respect to $P^{*}$ ). If the investor is unwilling or unable to put up the initial amount of capital required by this condition, but is ready to put up another amount $\nu<H_{0}$, then the arbitrage-free property of the market implies that the investor cannot replicate the claim $H$ in all possible scenarios; in other words, he cannot hedge the claim $H$ with probability 1.

In such a case, general results concerning the hedging, called quantile hedging, are obtained in the paper [4 if the price process is a semimartingale. The main aim of the quantile hedging is to maximize the probability of success, that is the probability of a successful hedge for a contingent claim $H$.

We consider the so-called mixed model of the price process with long-range dependence. This type of dependence can be seen in various fields of human activities, in particular in economics, finance markets, hydrophysics, and in telecommunication nets. In contrast to "pure" models involving only a single fractional Brownian model, the mixed model is arbitrage-free, at least in the case of the class of Markov self-financing strategies.

The set for which the probability of success is maximal is described in [1] for the mixed model of the price process. However, the structure of this set is rather complicated, and this causes a problem when calculating the probability of success. In its turn, the structure of the set is explained by a complicated form of the density $d P^{*} / d P$ that depends on the distribution of the corresponding processes on the whole interval $[0, T]$

2010 Mathematics Subject Classification. Primary 60G22, 91B24; Secondary 60G15.

Key words and phrases. Quantile hedging, fractional Brownian motion, mixed model, limit theorems, probability of success.

The second author is supported by the grant \#230804 "Multifractionality" of the European Commission. 
in the case of a mixed model (here, $P$ is the initial or objective measure). In particular, a lower estimate is obtained in [1] for the maximum probability of success in some cases of contingent claims; this estimate allows one to find a lower bound for the initial capital needed to hedge the contingent claim with a prescribed probability.

This paper is a continuation of [1]. We study convergence in probability of the sets of maximum probability of success in the problem of quantile hedging for the mixed model of the price process. The paper is organized as follows. Section 1 defines the mixed model of the price process. Section 2 describes a solution of the problem of quantile hedging used in the paper [1], proves some properties of the Volterra kernel determining the density $d P^{*} / d P$, and exhibits the set for which the probability of success is maximal. The convergence in probability of the sets of maximum probability of success is proved in Section 3.

1.1. A model of a market with long-range dependence. Let $(\Omega, \mathcal{F}, \mathrm{P})$ be a complete probability space equipped with a filtration $\left\{\mathcal{F}_{t}, t \geq 0\right\}$. Consider two independent processes defined on this probability space, namely a Wiener process $B=\left\{B_{t}, t \geq 0\right\}$ and a fractional Brownian motion $B^{H}=\left\{B_{t}^{H}, t \geq 0\right\}$ with Hurst index $H$. Recall that $B^{H}$ is a continuous Gaussian process starting from the origin and having zero expectation and the covariance function

$$
\mathrm{E} B_{t}^{H} B_{s}^{H}=\frac{1}{2}\left(s^{2 H}+t^{2 H}-|s-t|^{2 H}\right) .
$$

Consider the mixed model for which the price process is given by

$$
X_{t}=X_{0} \exp \left\{m t+\delta\left(B_{t}+\sigma B_{t}^{H}\right)\right\},
$$

where $X_{0}>0, m \in \mathbf{R}, \delta \in \mathbf{R} \backslash\{0\}$, and $\sigma>0$ are some constants.

Throughout the paper we restrict the consideration to the case of $H \in\left(\frac{3}{4} ; 1\right]$.

If $m=0$, then Theorem 4.2 of [2] implies that the process $M_{t}^{H, \sigma}=B_{t}+\sigma B_{t}^{H}$ is equivalent to some Wiener process. According to Theorem 1 of [3] (see also Theorem 4.15 of [2]) there exists a unique real-valued Volterra kernel

$$
h \in \mathbb{L}_{2}(0 \leq s<t \leq T)
$$

such that

$$
W_{t}=M_{t}^{H, \sigma}-\int_{0}^{t} \int_{0}^{s} h(s, u) M_{u}^{H, \sigma} d s
$$

is a Wiener process on $(\Omega, \mathcal{F}, \mathrm{P})$. Moreover,

$$
M_{t}^{H, \sigma}=W_{t}-\int_{0}^{t} \int_{0}^{s} r_{\sigma}(s, u) d W_{u} d s,
$$

where $W$ is a Wiener process with respect to the measure $\mathrm{P}$ and where

$$
r_{\sigma} \in \mathbb{L}_{2}(0 \leq s<t \leq T)
$$

is the inverse resolvent kernel for $h$ that is a unique solution of the equation

(3) $r_{\sigma}(t, s)+\int_{0}^{s} r_{\sigma}(t, x) r_{\sigma}(s, x) d x=\sigma^{2} H(2 H-1) \cdot(t-s)^{2 H-2}, \quad 0 \leq s<t \leq T$,

such that

$$
\int_{0}^{T} \int_{0}^{s}\left(r_{\sigma}(s, u)\right)^{2} d u d s<\infty .
$$

It is easy to check that the shifted process $(m / \sigma) t+B_{t}+\sigma B_{t}^{H}$ is also equivalent to a Wiener process. Indeed, one can consider two independent processes $(m / \sigma) t+B_{t}$ and 
$\sigma B_{t}^{H}$ and remove the shift for the process $(m / \sigma) t+B_{t}$. Thus $M_{t}^{H, \sigma}$ is a Wiener process with respect to the measure $P^{*}$ defined as follows:

$$
\begin{aligned}
\frac{d P^{*}}{d P}=\exp ( & -\left(\frac{m}{\delta}+\frac{1}{2} \delta\right) W_{t}+\int_{0}^{t} \int_{0}^{s} r_{\sigma}(s, u) d W_{u} d W_{s} \\
& \left.-\frac{1}{2} \int_{0}^{t}\left(\frac{m}{\delta}+\frac{1}{2} \delta-\int_{0}^{s} r_{\sigma}(s, u) d W_{u}\right)^{2} d s\right) .
\end{aligned}
$$

Remark 1.1. Neither the process $M_{t}^{H, \sigma}$ nor $X_{t}$ is a semimartingale with respect to the filtration generated by the family of processes $B_{t}$ and $B_{t}^{H}$; on the other hand, it is a semimartingale with respect to the natural filtration.

2. An APPlication OF the Föllmer-LEUKERT APPROACH TO THE MiXED MODEL OF THE PRICE PROCESS IN THE CASE OF A COMPLETE ARBITRAGE-FREE MARKET

2.1. Maximizing the probability of success. Recall that the problem of quantile hedging for the case of $\nu<H_{0}$ is to maximize the probability of the event $\left\{V_{T} \geq H\right\}$ in the class of all self-financing strategies $\xi$ with the initial capital $V_{0} \leq \nu$ that are feasible in the sense that the capital process

$$
V_{t}=V_{0}+\int_{0}^{t} \xi_{s} d X_{s}
$$

corresponding to the strategy $\xi$ is nonnegative almost surely for all $0 \leq t \leq T$. The general semimartingale approach to the solution of this problem uses the Neyman-Pearson lemma and is described in the paper [4. A modified Föllmer-Leukert approach is applied to the mixed Brownian-fractional Brownian model in [1]. We outline the solution of this problem for the general case and briefly discuss its features in the case of the mixed model.

A set $A=\left\{V_{T} \geq H\right\}$ is called the success set corresponding to the strategy $\xi$ (see (5) ). Consider the measure $Q^{*}$ defined by

$$
\frac{d Q^{*}}{d P^{*}}=\frac{H}{H_{0}}
$$

The optimization problem is to maximize the probability $\mathrm{P}(A)$ with respect to all $\mathcal{F}_{T}$ measurable sets $A$ such that

$$
\frac{V_{0}}{H_{0}}=\frac{\mathrm{E}_{P^{*}}\left(H I_{A}\right)}{H_{0}}=\mathrm{E}_{Q^{*}}\left(I_{A}\right) \leq \frac{\nu}{H_{0}} .
$$

Let

Assume that

$$
\bar{a}=\inf \left\{a: Q^{*}\left(\frac{d P}{d P^{*}}>a \cdot H\right) \leq \frac{\nu}{H_{0}}\right\} .
$$

$$
Q^{*}\left(\frac{d P}{d P^{*}}=\bar{a} \cdot H\right)=0 .
$$

According to the Neyman-Pearson lemma, the set

$$
A=A_{\bar{a}}:=\left\{\frac{d P}{d P^{*}}>\bar{a} \cdot H\right\}
$$

is optimal.

Due to the choice of $\bar{a}$, given the initial capital $\nu$, there exists a strategy that hedges the contingent claim $\bar{H}=H I_{A_{\bar{a}}}$ with probability one. Thus the same strategy allows one to hedge the claim $H$ with probability $\mathrm{P}\left(A_{\bar{a}}\right)$. This is a strategy that maximizes the probability $\mathrm{P}\left(V_{T} \geq H\right)$. 
2.2. Evaluating the maximum probability of success. Consider one of the simplest cases where the claim $H$ depends on the price of an asset at the terminal moment only, that is, $H=H_{T}=f\left(X_{T}\right)$. Using the Neyman-Pearson lemma, the problem is to find a constant $\bar{a}$ such that the probability $\mathrm{P}$ of the set

$$
A=A_{a}:=\left\{\frac{d P}{d P^{*}}>a \cdot H\right\}
$$

is maximal provided $Q^{*}(A) \leq \nu / H_{0}$. Taking into account expression (4) for the density, the set $A$ can be rewritten as follows:

$$
\begin{aligned}
A=\{\exp ( & \left(\frac{m}{\delta}+\frac{1}{2} \delta\right) W_{T}-\int_{0}^{T} \int_{0}^{s} r_{\sigma}(s, u) d W_{u} d W_{s} \\
& \left.\left.+\frac{1}{2} \int_{0}^{T}\left(\frac{m}{\delta}+\frac{1}{2} \delta-\int_{0}^{s} r_{\sigma}(s, u) d W_{u}\right)^{2} d s\right)>a \cdot f\left(X_{T}\right)\right\} .
\end{aligned}
$$

This set depends on the process $W=\left\{W_{t}, t \geq 0\right\}$ in the whole interval $[0, T]$ and thus the evaluation of the probabilities $Q^{*}(A)$ and $\mathrm{P}(A)$ is rather complicated. Therefore the approach of [4] is not easy to apply even for simplest contingent claims $f\left(X_{T}\right)$ (for European call options, for example).

Nevertheless the problem of stability of the probability of the event $A$ can be solved without having calculated $\mathrm{P}(A)$ if the parameters $m, \delta$, and $\sigma$ vary continuously.

2.3. Properties of the kernel $r_{\sigma}$. First we consider some properties of the kernel $r_{\sigma}$ that will be used below in the proof of limit theorems concerning the stability of $\mathrm{P}(A)$ in the case where $r_{\sigma}$ satisfies condition (3).

Lemma 2.1. The kernel $r_{\sigma}$ is such that

$$
\left|r_{\sigma}(t, s)\right|<\sigma^{2} H(2 H-1)(t-s)^{2 H-2}+C_{T, H, \sigma},
$$

where

$$
\begin{gathered}
C_{T, H, \sigma}=2 H^{2}(2 H-1)^{2} \frac{T^{4 H-3}}{4 H-3} \sigma^{4} \exp \left(2 C_{T, \sigma}\right), \\
C_{T, \sigma}=\int_{0}^{T} \int_{0}^{s}\left(r_{\sigma}(s, u)\right)^{2} d u d s<\infty .
\end{gathered}
$$

Proof. It is shown in Lemma 2.2 of [7] that

$$
\int_{0}^{s} r_{\sigma}^{2}(t, x) d x \leq C_{T, H, \sigma}, \quad 0 \leq s \leq t \leq T .
$$

Hence

$$
\left|\int_{0}^{s} r_{\sigma}(t, x) r_{\sigma}(s, x) d x\right| \leq \sqrt{\int_{0}^{s} r_{\sigma}^{2}(t, x) d x \int_{0}^{s} r_{\sigma}^{2}(s, x) d x} \leq C_{T, H, \sigma} .
$$

The rest of the proof follows from (3) and (12).

Lemma 2.2. The kernel $r_{\sigma}$ is continuous with respect to the first argument.

Proof. Let $t^{\prime} \rightarrow t$ and put $\varepsilon=(t-s) / 2$. Since $t>s$, we restrict the consideration to the case $t^{\prime}>s+\varepsilon$ without loss of generality.

Equation (3) implies that

$$
\begin{aligned}
\left|r_{\sigma}\left(t^{\prime}, s\right)-r_{\sigma}(t, s)\right| \leq & H(2 H-1) \sigma^{2}\left|\left(t^{\prime}-s\right)^{2 H-2}-(t-s)^{2 H-2}\right| \\
& +\int_{0}^{s}\left|r_{\sigma}(s, x)\right| \cdot\left|r_{\sigma}\left(t^{\prime}, x\right)-r_{\sigma}(t, x)\right| d x
\end{aligned}
$$


To estimate the first term on the right hand side of inequality (13), we use the Lagrange mean value theorem and take into account the inequality $\varepsilon<\min \left(t^{\prime}-s, t-s\right)$ :

$$
\left|\left(t^{\prime}-s\right)^{2 H-2}-(t-s)^{2 H-2}\right| \leq\left(t^{\prime}-t\right) \cdot(2-2 H) \cdot \varepsilon^{2 H-3} .
$$

Thus

where

$$
H(2 H-1) \sigma^{2}\left|\left(t^{\prime}-s\right)^{2 H-2}-(t-s)^{2 H-2}\right| \leq A_{H, \sigma, \varepsilon} \cdot\left|t^{\prime}-t\right|,
$$

$$
A_{H, \sigma, \varepsilon}=H(2 H-1) \sigma^{2}(2-2 H) \cdot \varepsilon^{2 H-3} .
$$

Putting $\Delta_{\sigma}\left(s, t^{\prime}, t\right)=\left|r_{\sigma}\left(t^{\prime}, s\right)-r_{\sigma}(t, s)\right|$, we rewrite inequality (13) as follows:

$$
\Delta_{\sigma}\left(s, t^{\prime}, t\right) \leq A_{H, \sigma, \varepsilon} \cdot\left|t^{\prime}-t\right|+\int_{0}^{s}\left|r_{\sigma}(s, x)\right| \cdot \Delta_{\sigma}\left(x, t^{\prime}, t\right) d x
$$

whence

$$
\begin{aligned}
\left(\Delta_{\sigma}\left(s, t^{\prime}, t\right)\right)^{2} & \leq 2 A_{H, \sigma, \varepsilon}^{2} \cdot\left|t^{\prime}-t\right|^{2}+2\left(\int_{0}^{s}\left|r_{\sigma}(s, x)\right| \cdot \Delta_{\sigma}\left(x, t^{\prime}, t\right) d x\right)^{2} \\
& \leq 2 A_{H, \sigma, \varepsilon}^{2} \cdot\left|t^{\prime}-t\right|^{2}+2 \int_{0}^{s} r_{\sigma}^{2}(s, x) d x \cdot \int_{0}^{s}\left(\Delta_{\sigma}\left(x, t^{\prime}, t\right)\right)^{2} d x \\
& \leq 2 A_{H, \sigma, \varepsilon}^{2} \cdot\left|t^{\prime}-t\right|^{2}+2 C_{T, H, \sigma} \cdot \int_{0}^{s}\left(\Delta_{\sigma}\left(x, t^{\prime}, t\right)\right)^{2} d x .
\end{aligned}
$$

Finally, the Gronwall inequality implies that

$$
\begin{aligned}
\left(\Delta_{\sigma}\left(s, t^{\prime}, t\right)\right)^{2} & \leq 2 A_{H, \sigma, \varepsilon}^{2} \cdot\left|t^{\prime}-t\right|^{2} \cdot \exp \left(2 C_{T, H, \sigma} \cdot s\right) \\
& \leq 2 A_{H, \sigma, \varepsilon}^{2} \cdot\left|t^{\prime}-t\right|^{2} \cdot \exp \left(2 C_{T, H, \sigma} \cdot T\right) .
\end{aligned}
$$

Inequality (16) implies the continuity of the function $r_{\sigma}(t, s)$ with respect to the first argument.

Remark 2.1. Bound (16) implies that the function $r_{\sigma}(t, s)$ is uniformly continuous with respect to the first argument.

Lemma 2.3. The kernel $r_{\sigma}$ is continuous with respect to the second argument.

Proof. First we prove the right continuity. Let $s^{\prime} \rightarrow s+$ and

$$
s<s^{\prime}<t \text {. }
$$

Then

$$
\begin{aligned}
r_{\sigma}\left(t, s^{\prime}\right)-r_{\sigma}(t, s)= & H(2 H-1) \sigma^{2}\left|\left(t-s^{\prime}\right)^{2 H-2}-(t-s)^{2 H-2}\right| \\
& -\int_{0}^{s} r_{\sigma}(t, x) \cdot\left(r_{\sigma}\left(s^{\prime}, x\right)-r_{\sigma}(s, x)\right) d x \\
& -\int_{s}^{s^{\prime}} r_{\sigma}(t, x) r_{\sigma}\left(s^{\prime}, x\right) d x .
\end{aligned}
$$

This implies that

$$
\begin{aligned}
\left(r_{\sigma}\left(t, s^{\prime}\right)-r_{\sigma}(t, s)\right)^{2} \leq & 3 H^{2}(2 H-1)^{2} \sigma^{4}\left(\left(t-s^{\prime}\right)^{2 H-2}-(t-s)^{2 H-2}\right)^{2} \\
& +3 \int_{0}^{s} r_{\sigma}^{2}(t, x) d x \cdot \int_{0}^{s}\left(r_{\sigma}\left(s^{\prime}, x\right)-r_{\sigma}(s, x)\right)^{2} d x \\
& +3 \int_{s}^{s^{\prime}} r_{\sigma}^{2}(t, x) d x \cdot \int_{s}^{s^{\prime}} r_{\sigma}^{2}\left(s^{\prime}, x\right) d x .
\end{aligned}
$$

The first term on the right hand side of inequality (18) approaches 0 as $s^{\prime} \rightarrow s+$. To estimate the second term, we use Lemma 2.2 and prove that $r_{\sigma}\left(s^{\prime}, x\right)-r_{\sigma}(s, x) \rightarrow 0$ as 
$s^{\prime} \rightarrow s+$. We are going to apply the Lebesgue dominated convergence theorem with the integrable upper bound

$$
\begin{aligned}
\left(r_{\sigma}\left(s^{\prime}, x\right)-r_{\sigma}(s, x)\right)^{2} \leq & 2 H^{2}(2 H-1)^{2} \sigma^{4}\left(\left(s^{\prime}-x\right)^{2 H-2}-(s-x)^{2 H-2}\right)^{2} \\
& +2 \int_{0}^{x} r_{\sigma}^{2}(x, y) d y \cdot \int_{0}^{x}\left(r_{\sigma}\left(s^{\prime}, y\right)-r_{\sigma}(s, y)\right)^{2} d y \\
\leq & 2 H^{2}(2 H-1)^{2} \sigma^{4} \cdot(s-x)^{4 H-4}+8 C_{T, H, \sigma}^{2} .
\end{aligned}
$$

The latter bounds hold in view of

$$
\int_{0}^{x} r_{\sigma}^{2}(x, y) d y \leq C_{T, H, \sigma}
$$

and

$$
\begin{aligned}
\int_{0}^{x}\left(r_{\sigma}\left(s^{\prime}, y\right)-r_{\sigma}(s, y)\right)^{2} d y & \leq 2 \int_{0}^{x} r_{\sigma}^{2}\left(s^{\prime}, y\right) d y+2 \int_{0}^{x} r_{\sigma}^{2}(s, y) d y \\
& \leq 2 \int_{0}^{s^{\prime}} r_{\sigma}^{2}\left(s^{\prime}, y\right) d y+2 \int_{0}^{s} r_{\sigma}^{2}(s, y) d y \leq 4 C_{T, H, \sigma} .
\end{aligned}
$$

Now the Lebesgue dominated convergence theorem implies that

$$
\int_{0}^{s}\left(r_{\sigma}\left(s^{\prime}, x\right)-r_{\sigma}(s, x)\right)^{2} d x \rightarrow 0, \quad s^{\prime} \rightarrow s+
$$

and thus the second term approaches 0 as $s^{\prime} \rightarrow s+$.

Then we estimate the third term on the right hand side of inequality (18) by using equality (3):

$$
\begin{aligned}
\int_{s}^{s^{\prime}} r_{\sigma}^{2}\left(s^{\prime}, x\right) d x \leq & 2 \int_{s}^{s^{\prime}} H^{2}(2 H-1)^{2} \sigma^{4}\left(s^{\prime}-x\right)^{4 H-4} d x \\
& +2 \int_{s}^{s^{\prime}}\left(\int_{0}^{x} r_{\sigma}^{2}(x, y) d y\right) \cdot\left(\int_{0}^{x} r_{\sigma}^{2}\left(s^{\prime}, y\right) d y\right) d x \\
\leq & 2 H^{2}(2 H-1)^{2} \sigma^{4} \cdot \frac{\left(s^{\prime}-s\right)^{4 H-3}}{4 H-3}+2 C_{T, H, \sigma}^{2}\left(s^{\prime}-s\right) \rightarrow 0 \\
s^{\prime} \rightarrow s+ &
\end{aligned}
$$

since $H>\frac{3}{4}$. This completes the proof of the right continuity.

Now we prove that the kernel $r_{\sigma}$ is left continuous with respect to the second argument. Approaching the limit as $s^{\prime} \rightarrow s-$, equality (17) becomes of the form

$$
\begin{aligned}
r_{\sigma}\left(t, s^{\prime}\right)-r_{\sigma}(t, s)= & H(2 H-1) \sigma^{2}\left|\left(t-s^{\prime}\right)^{2 H-2}-(t-s)^{2 H-2}\right| \\
& -\int_{0}^{s} \mathbf{1}_{\left\{x<s^{\prime}\right\}} \cdot r_{\sigma}(t, x) \cdot\left(r_{\sigma}\left(s^{\prime}, x\right)-r_{\sigma}(s, x)\right) d x \\
& +\int_{s^{\prime}}^{s} r_{\sigma}(t, x) r_{\sigma}(s, x) d x .
\end{aligned}
$$

Equalities (21) and (17) differ by the second term on their right hand sides. Note that the proof that the first and third terms on the right hand side of (21) approach 0 as $s^{\prime} \rightarrow s-$ is the same as in the case of (17). Therefore we only need to prove that the second term approaches 0 as $s^{\prime} \rightarrow s-$. Fix $\theta \in(0, s)$ and represent the second term as 
the sum

$$
\begin{aligned}
\int_{0}^{s} \mathbf{1}_{\left\{x<s^{\prime}\right\}} \cdot r_{\sigma}(t, x) \cdot\left(r_{\sigma}\left(s^{\prime}, x\right)-r_{\sigma}(s, x)\right) d x \\
=\int_{0}^{s-\theta} \mathbf{1}_{\left\{x<s^{\prime}\right\}} \cdot r_{\sigma}(t, x) \cdot\left(r_{\sigma}\left(s^{\prime}, x\right)-r_{\sigma}(s, x)\right) d x \\
\quad+\int_{s-\theta}^{s} \mathbf{1}_{\left\{x<s^{\prime}\right\}} \cdot r_{\sigma}(t, x) \cdot\left(r_{\sigma}\left(s^{\prime}, x\right)-r_{\sigma}(s, x)\right) d x .
\end{aligned}
$$

Now we check that, for all $\varepsilon>0$, one can choose $\theta \in(0, s)$ such that none of the terms exceeds $\varepsilon / 2$. Since

$$
\begin{aligned}
& \left(\int_{s-\theta}^{s} \mathbf{1}_{\left\{x<s^{\prime}\right\}} \cdot r_{\sigma}(t, x) \cdot\left(r_{\sigma}\left(s^{\prime}, x\right)-r_{\sigma}(s, x)\right) d x\right)^{2} \\
& \quad \leq \int_{s-\theta}^{s} r_{\sigma}^{2}(t, x) d x \cdot \int_{s-\theta}^{s} \mathbf{1}_{\left\{x<s^{\prime}\right\}}\left(2 r_{\sigma}^{2}\left(s^{\prime}, x\right)+2 r_{\sigma}^{2}(s, x)\right) d x \\
& \quad \leq \int_{s-\theta}^{s} r_{\sigma}^{2}(t, x) d x \cdot\left(2 \int_{0}^{s^{\prime}} r_{\sigma}^{2}\left(s^{\prime}, x\right) d x+2 \int_{0}^{s} r_{\sigma}^{2}(s, x) d x\right) \\
& \quad \leq \int_{s-\theta}^{s} r_{\sigma}^{2}(t, x) d x \cdot 4 C_{T, H, \sigma},
\end{aligned}
$$

a number $\theta$ can be chosen so that the inequality

$$
\int_{s-\theta}^{s} r_{\sigma}^{2}(t, x) d x \cdot 4 C_{T, H, \sigma}<\frac{\varepsilon^{2}}{4}
$$

holds. For an arbitrary $\theta \in(0, s)$, the expression

$$
\int_{0}^{s-\theta} \mathbf{1}_{\left\{x<s^{\prime}\right\}} \cdot r_{\sigma}(t, x) \cdot\left(r_{\sigma}\left(s^{\prime}, x\right)-r_{\sigma}(s, x)\right) d x
$$

approaches 0 as $s^{\prime} \rightarrow s-$ and thus eventually becomes less than $\varepsilon / 2$, since the expression under the integral sign approaches 0 . If $s^{\prime}>s-\theta / 2$, then $s^{\prime}-x>\theta / 2$ and therefore the majorizing function in the Lebesgue dominated convergence theorem is given by

$$
\begin{aligned}
\left(r_{\sigma}\left(s^{\prime}, x\right)-r_{\sigma}(s, x)\right)^{2} \leq & 2 H^{2}(2 H-1)^{2} \sigma^{4}\left(\left(s^{\prime}-x\right)^{2 H-2}-(s-x)^{2 H-2}\right)^{2} \\
& +2 \int_{0}^{x} r_{\sigma}^{2}(x, y) d y \cdot \int_{0}^{x}\left(r_{\sigma}\left(s^{\prime}, y\right)-r_{\sigma}(s, y)\right)^{2} d y \\
\leq & 2 H^{2}(2 H-1)^{2} \sigma^{4} \cdot\left(\frac{\theta}{2}\right)^{4 H-4}+8 C_{T, H, \sigma}^{2} .
\end{aligned}
$$

Therefore the kernel $r_{\sigma}$ is continuous with respect to the second argument.

Lemma 2.4. The kernel $r_{\sigma}=r_{\sigma}(t, s)$ is continuous with respect to $\sigma$ for all fixed $s$ and $t$ such that $0 \leq s<t<T$.

Proof. For fixed $0 \leq s<t<T$, we show that $r_{\sigma_{n}}(t, s) \rightarrow r_{\sigma}(t, s)$ as $\sigma_{n} \rightarrow \sigma$. Put $\alpha=H-\frac{1}{2}$. Since $t\left(\sigma_{n} / \sigma\right)^{1 / \alpha} \leq T$ for sufficiently large $n$, the self-similarity proved in Lemma 2.1 of [7] implies that

$$
r_{\sigma_{n}}(t, s)=\left(\frac{\sigma_{n}}{\sigma}\right)^{1 / \alpha} r_{\sigma}\left(t\left(\frac{\sigma_{n}}{\sigma}\right)^{1 / \alpha}, s\left(\frac{\sigma_{n}}{\sigma}\right)^{1 / \alpha}\right) .
$$

Recall that the property of self-similarity means that $r_{\sigma}(t, s)=\sigma^{1 / \alpha} r_{1}\left(\sigma^{1 / \alpha} t, \sigma^{1 / \alpha} s\right)$ for $0 \leq s<t \leq T \wedge T \sigma^{-1 / \alpha}$. 
Note that $\left(\sigma_{n} / \sigma\right)^{1 / \alpha} \rightarrow 1$ as $n \rightarrow \infty$, whence it follows that we only need to prove that

$$
r_{\sigma}\left(t\left(\frac{\sigma_{n}}{\sigma}\right)^{1 / \alpha}, s\left(\frac{\sigma_{n}}{\sigma}\right)^{1 / \alpha}\right) \rightarrow r_{\sigma}(t, s), \quad \sigma_{n} \rightarrow \sigma .
$$

We have

$$
\begin{aligned}
\mid r_{\sigma}(t, s) & -r_{\sigma}\left(t\left(\frac{\sigma_{n}}{\sigma}\right)^{1 / \alpha}, s\left(\frac{\sigma_{n}}{\sigma}\right)^{1 / \alpha}\right) \mid \\
\leq & \left|r_{\sigma}(t, s)-r_{\sigma}\left(t, s\left(\frac{\sigma_{n}}{\sigma}\right)^{1 / \alpha}\right)\right| \\
& +\left|r_{\sigma}\left(t, s\left(\frac{\sigma_{n}}{\sigma}\right)^{1 / \alpha}\right)-r_{\sigma}\left(t\left(\frac{\sigma_{n}}{\sigma}\right)^{1 / \alpha}, s\left(\frac{\sigma_{n}}{\sigma}\right)^{1 / \alpha}\right)\right| .
\end{aligned}
$$

According to Lemma 2.3, the kernel $r_{\sigma}(t, s)$ is continuous with respect to the second argument and thus

$$
\left|r_{\sigma}(t, s)-r_{\sigma}\left(t, s\left(\frac{\sigma_{n}}{\sigma}\right)^{1 / \alpha}\right)\right| \rightarrow 0, \quad n \rightarrow \infty .
$$

Note that the second term on the right hand side of inequality (25) can be written as follows:

$$
\Delta_{\sigma}\left(s\left(\frac{\sigma_{n}}{\sigma}\right)^{1 / \alpha}, t\left(\frac{\sigma_{n}}{\sigma}\right)^{1 / \alpha}, t\right)
$$

and that inequality (16) in Lemma 2.2 holds for all numbers $s, t^{\prime}$, and $t$ such that $\varepsilon<\min \left(t-s, t^{\prime}-s\right)$.

Since $\left(\sigma_{n} / \sigma\right)^{1 / \alpha} \rightarrow 1, n \rightarrow \infty$, the inequality

$$
\varepsilon=\frac{t-s}{2}<\min \left(t-s\left(\frac{\sigma_{n}}{\sigma}\right)^{1 / \alpha}, t\left(\frac{\sigma_{n}}{\sigma}\right)^{1 / \alpha}-s\left(\frac{\sigma_{n}}{\sigma}\right)^{1 / \alpha}\right)
$$

holds for all sufficiently large $n$. Hence inequality (16) and Remark 2.1 imply that

$$
\Delta_{\sigma}\left(s\left(\frac{\sigma_{n}}{\sigma}\right)^{1 / \alpha}, t\left(\frac{\sigma_{n}}{\sigma}\right)^{1 / \alpha}, t\right) \rightarrow 0, \quad n \rightarrow \infty,
$$

which means that

$$
\left|r_{\sigma}\left(t, s\left(\frac{\sigma_{n}}{\sigma}\right)^{1 / \alpha}\right)-r_{\sigma}\left(t\left(\frac{\sigma_{n}}{\sigma}\right)^{1 / \alpha}, s\left(\frac{\sigma_{n}}{\sigma}\right)^{1 / \alpha}\right)\right| \rightarrow 0, \quad n \rightarrow \infty .
$$

2.4. Maximal estimates for Gaussian stochastic processes. Consider a method to estimate the supremum of Gaussian stochastic processes needed later in the proof of the limit theorems.

Let

$$
U(x)=\exp \left\{\frac{x^{2}}{2}\right\}-1 .
$$

Definition 2.1. The space of random variables $\xi$ such that there exists a constant $C_{\xi}>0$ for which

$$
\mathrm{E} U\left(\frac{\xi}{C_{\xi}}\right)<\infty
$$

is called the Orlicz space $L_{U}(\Omega)$ generated by the function $U(x)$.

Theorem 2.1 ([5]). The Orlicz space is a Banach space with respect to the Luxemburg metric

$$
\|\xi\|_{U}=\inf \left\{r>0: \operatorname{E} \exp \left\{\frac{\xi^{2}}{r^{2}}\right\} \leq 2\right\} .
$$


In particular, if $\xi=N\left(0, \sigma^{2}\right)$, then $\xi$ belongs to the Orlicz space $L_{U}(\Omega)$, where $U(x)$ is defined by (26). Moreover, $\|\xi\|_{U}=2 \sigma / \sqrt{3}$.

Proposition 2.1 (Lemma 2.3.3 of [5]). If $\xi \in L_{U}(\Omega)$, where $U$ is defined by (26) , then $\mathrm{E}|\xi| \leq \sqrt{2}\|\xi\|_{U}$

Let $\mathfrak{X}=\{\mathfrak{X}(t), t \in \mathfrak{T}\}$ be a stochastic process belonging to the space $L_{U}(\Omega)$, where $U$ is defined by (26), let $\rho(t, s)=\|\mathfrak{X}(t)-\mathfrak{X}(s)\|_{U}$, and let $\mathfrak{X}(t)$ be a separable process on $(\mathfrak{T}, \rho)$. Then Theorem 3.3.1 of [5] implies that

$$
\left\|\sup _{t \in \mathfrak{T}} \mathfrak{X}(t)\right\|_{U} \leq \inf _{t \in \mathfrak{T}}\|\mathfrak{X}(t)\|_{U}+\frac{1}{\theta(1-\theta)} \int_{0}^{\varepsilon_{0} \theta} \varkappa(N(\varepsilon)) d \varepsilon,
$$

provided the integral on the right hand side of (27) converges. Here $\theta$ is an arbitrary number in the interval $(0,1), \varepsilon_{0}=\sup _{t, s \in \mathfrak{T}} \rho(t, s), N(u)$ is the metric capacity of $(\mathfrak{T}, \rho)$, that is, the least number of balls of radius $u$ covering the space $(\mathfrak{T}, \rho)$, and where $\varkappa(n)$ is the majorizing characteristics. Note that

$$
\varkappa(n)=e(2 \ln (n+1))^{1 / 2}
$$

in the case of (26) (see Example 2.3.2 in [5]).

Now let $\mathfrak{T}=[a, b]$ and $\sup _{|t-s| \leq h}\|\mathfrak{X}(t)-\mathfrak{X}(s)\| \leq \sigma(h)$, where $\sigma(h)$ is a continuous nondecreasing function such that $\sigma(0)=0$. Since

$$
N(u) \leq \frac{b-a}{2 \sigma^{(-1)}(u)}+1
$$

we obtain

$$
\begin{aligned}
\left\|\sup _{t \in T} \mathfrak{X}(t)\right\|_{U} & \leq \inf _{t \in T}\|\mathfrak{X}(t)\|_{U}+\frac{1}{\theta(1-\theta)} \int_{0}^{\varepsilon_{0} \theta} \varkappa\left(\frac{b-a}{2 \sigma^{(-1)}(u)}+1\right) d u \\
& =\inf _{t \in T}\|\mathfrak{X}(t)\|_{U}+\frac{e \sqrt{2}}{\theta(1-\theta)} \int_{0}^{\varepsilon_{0} \theta}\left(\ln \left(\frac{b-a}{2 \sigma^{(-1)}(u)}+2\right)\right)^{1 / 2} d u .
\end{aligned}
$$

The latter integral converges in particular if

$$
\sigma(h)=C h^{\rho}
$$

for some $\rho>0$.

2.5. Estimates for an increment of the integral with a kernel. Let $\{W(t), t \geq 0\}$ be a Wiener process. It is obvious that the integral $\int_{0}^{t} r_{\sigma}(t, u) d W(u)$ exists for all $t>0$ and is a Gaussian stochastic process with respect to $t$.

Lemma 2.5. Let $H>\frac{3}{4}$ and let $Z(t)=\int_{0}^{t} r_{\sigma}(t, u) d W(u)$. Then $Z$ is a Gaussian process and

$$
\mathrm{E}|Z(t)-Z(s)|^{2}<C \cdot|t-s|^{2 H-3 / 2} .
$$

Proof. Without loss of generality, we assume that $t>s$. Since the random variables

$$
\int_{0}^{s}\left(r_{\sigma}(t, u)-r_{\sigma}(s, u)\right) d W(u) \quad \text { and } \quad \int_{s}^{t} r_{\sigma}(t, u) d W(u)
$$

are independent and have zero expectations, we get

$$
\mathrm{E}|Z(t)-Z(s)|^{2}=\int_{0}^{s}\left(r_{\sigma}(t, u)-r_{\sigma}(s, u)\right)^{2} d u+\int_{s}^{t} r_{\sigma}^{2}(t, u) d u
$$


Each of the terms will be estimated separately. Positive constants in the proof below are denoted by $C$. We do not indicate the precise values of the constants if they do not matter for the reasoning. To estimate the second term, we use inequality (11):

$$
\left|r_{\sigma}(t, u)\right|<C \cdot(t-u)^{2 H-2}+C \leq C \cdot(t-u)^{2 H-2} .
$$

Then

$$
\int_{s}^{t} r_{\sigma}^{2}(t, u) d u \leq \int_{s}^{t} C(t-u)^{4 H-4} d u=C \cdot(t-s)^{4 H-3} .
$$

We recall that $H \in\left(\frac{3}{4}, 1\right)$, whence $4 H-3>0$.

Now we estimate the first term $\int_{0}^{s}\left(r_{\sigma}(t, u)-r_{\sigma}(s, u)\right)^{2} d u$. Using the Lagrange mean value theorem we obtain

$$
\left|(s-u)^{2 H-2}-(t-u)^{2 H-2}\right| \leq(2-2 H)(t-s)(s-u)^{2 H-3} .
$$

On the other hand,

$$
\left|(s-u)^{2 H-2}-(t-u)^{2 H-2}\right| \leq(s-u)^{2 H-2} .
$$

Thus

$$
\begin{aligned}
\left|(s-u)^{2 H-2}-(t-u)^{2 H-2}\right| & \leq C(s-u)^{(2 H-2) \gamma}(s-u)^{(2 H-3)(1-\gamma)}(t-s)^{1-\gamma} \\
& =C(t-s)^{1-\gamma}(s-u)^{2 H-3+\gamma}
\end{aligned}
$$

for all $\gamma \in(0,1)$. Let $\gamma=\frac{7}{4}-H$; since $H \in\left(\frac{3}{4}, 1\right)$, we have $\gamma \in(0,1)$ and $2 H-3+\gamma>-\frac{1}{2}$.

Similarly to the proof of Lemma 2.2, we use inequality (3) and show that

$$
\begin{aligned}
\left(\Delta_{\sigma}(u, t, s)\right)^{2} \leq & 2\left(H(2 H-1) \sigma^{2}\left|(t-u)^{2 H-2}-(s-u)^{2 H-2}\right|\right)^{2} \\
& +2 C_{T, H, \sigma} \int_{0}^{u} \Delta_{\sigma}^{2}(x, t, s) d x .
\end{aligned}
$$

Now inequality (31) implies that

$$
\begin{aligned}
\left|(s-v)^{2 H-2}-(t-v)^{2 H-2}\right| & \leq C(t-s)^{1-\gamma}(s-v)^{2 H-3+\gamma} \\
& \leq C(t-s)^{1-\gamma}(s-u)^{2 H-3+\gamma}
\end{aligned}
$$

for all $v \in(0, u]$.

Then we obtain from inequalities (32) and (33) that

$$
\begin{aligned}
\left(\Delta_{\sigma}(v, t, s)\right)^{2} \leq & 2\left(H(2 H-1) \sigma^{2}\left|(t-v)^{2 H-2}-(s-v)^{2 H-2}\right|\right)^{2} \\
& +2 C_{T, H, \sigma} \int_{0}^{v} \Delta_{\sigma}^{2}(x, t, s) d x \\
\leq & C(t-s)^{2-2 \gamma}(s-u)^{4 H-6+2 \gamma}+2 C_{T, H, \sigma} \int_{0}^{v} \Delta_{\sigma}^{2}(x, t, s) d x .
\end{aligned}
$$

The expression $C(t-s)^{1-\gamma}(s-u)^{2 H-3+\gamma}$ in the latter inequality does not depend on $v$. Therefore

$$
\left(\Delta_{\sigma}(v, t, s)\right)^{2} \leq C(t-s)^{2-2 \gamma}(s-u)^{4 H-6+2 \gamma}
$$

by the Gronwall inequality. Since inequality (34) holds for all $v \in(0, u]$, we choose $v=u$ and conclude that

$$
\left(\Delta_{\sigma}(u, t, s)\right)^{2} \leq C(t-s)^{2-2 \gamma}(s-u)^{4 H-6+2 \gamma} .
$$


Note that $4 H-6+2 \gamma>-1$. Thus

$$
\begin{aligned}
\int_{0}^{s}\left(r_{\sigma}(t, u)-r_{\sigma}(s, u)\right)^{2} d u & \leq \int_{0}^{s} C(t-s)^{2-2 \gamma}(s-u)^{4 H-6+2 \gamma} d u \\
& \leq C(t-s)^{2-2 \gamma} .
\end{aligned}
$$

Hence

$$
\mathrm{E}|Z(t)-Z(s)|^{2}<C(t-s)^{4 H-3}+C(t-s)^{2-2 \gamma}<C|t-s|^{\beta},
$$

where $\beta=\min \{4 H-3 ; 2-2 \gamma\}=2 H-\frac{3}{2}$ and where $C>0$ is a constant.

\section{Convergence in probability of sets WITH MAXIMUM PROBABILITY OF SUCCESS}

Consider the case where the parameters $m, \delta$, and $\sigma$ of the process $X_{t}$ are varying, that is,

$$
X_{t}^{n}=X_{0} \exp \left\{m_{n} t+\delta_{n}\left(B_{t}+\sigma_{n} B_{t}^{H}\right)\right\}, \quad n \geq 1 .
$$

Generally speaking, the Wiener process in representation (2) is varying too and the set (10) of the maximum probability of success is of the form

$$
\begin{aligned}
A_{n}=\{\exp & \left(\left(\frac{m_{n}}{\delta_{n}}+\frac{1}{2} \delta_{n}\right) W_{T}^{n}-\int_{0}^{T} \int_{0}^{s} r_{\sigma_{n}}(s, u) d W_{u}^{n} d W_{s}^{n}\right. \\
& \left.\left.+\frac{1}{2} \int_{0}^{T}\left(\frac{m_{n}}{\delta_{n}}+\frac{1}{2} \delta_{n}-\int_{0}^{s} r_{\sigma_{n}}(s, u) d W_{u}^{n}\right)^{2} d s\right)>a \cdot f\left(X_{T}\right)\right\}
\end{aligned}
$$

Next we study the question of convergence in probability of the sets $A_{n}$ as $n \rightarrow \infty$ if all the parameters converge to some limit values.

3.1. Convergence in probability of the sets $A_{n}$. This convergence follows from a result of $[6]$. First we introduce some notation. Let $\left(\Omega^{n}, \mathcal{F}^{n},\left(\mathcal{F}_{t}^{n}\right)_{t \in[0, T]}, P^{n}\right)$ be a stochastic basis for all $n$. Fix a countable set $\mathbf{T}$ everywhere dense in $[0, T]$ and such that $0 \in \mathbf{T}$.

We denote by $L_{\mathbf{T}}$ the class of all sequences

$$
\alpha_{k}=\left\{0=t_{0 k}<t_{1 k}<\cdots<t_{l_{k} k}<T\right\}
$$

of finite partitions of the interval $[0, T]$ satisfying the following two conditions:

(1) $\alpha_{k} \subseteq \alpha_{k+1} \subseteq \mathbf{T}$

(2) for all $t \in \mathbf{T}$, there exists $k(t)$ such that $t \in \alpha_{k}$ for $k>k(t)$.

Put

$$
\begin{gathered}
\Delta_{i k} x:=x\left(t_{i k}\right)-x\left(t_{i-1 k}\right), \\
\omega_{i k} x=\sup _{t_{i-1} \leq s<t \leq t_{i k}}|x(t)-x(s)| \\
\mathcal{T}\left(\mathcal{F}^{n}\right)=\left\{\tau: 0 \leq \tau \leq T \text { and } \tau \text { is an } \mathcal{F}^{n} \text { stopping time }\right\} .
\end{gathered}
$$

The symbol " $\stackrel{d}{\rightarrow}$ " denotes weak convergence of finite-dimensional distributions of stochastic processes, while the symbol " $\Rightarrow$ " stands for weak convergence of measures generated by the corresponding stochastic processes.

Let

$$
\left\{X_{n}(t), \mathcal{F}_{t}^{n}, t \in[0, T], n \in \mathbf{Z}_{+}\right\}=\left\{X_{n}^{1}(t), X_{n}^{2}(t), \ldots, X_{n}^{d}(t), \mathcal{F}_{t}^{n}, t \in[0, T], n \in \mathbf{Z}_{+}\right\}
$$


be a sequence of $d$-dimensional semimartingales with components admitting the following decomposition:

$$
X_{n}^{j}(t)=X_{n}^{j}(0)+M_{n}^{j}(t)+B_{n}^{j}(t), \quad 1 \leq j \leq d,
$$

where $\left\{M_{n}^{j}(t), \mathcal{F}_{t}^{n}, t \in[0, T], n \in \mathbf{Z}_{+}\right\}$is a sequence of square-integrable martingales with continuous trajectories and where $\left\{B_{n}^{j}(t), t \in[0, T], n \in \mathbf{Z}_{+}\right\}$is a sequence of continuous processes of bounded variation.

Let $\left\{\mu_{n}^{j}(t):=\left\langle M_{n}^{j}\right\rangle(t)\right.$ be the quadratic characteristics of the corresponding martingales. Also let

$$
\left\{\xi_{n}^{j}(t), \mathcal{F}_{t}^{n}, t \in[0, T], n \in \mathbf{Z}_{+}, 1 \leq j \leq d\right\}
$$

be a sequence of $\mathcal{F}^{n}$-predictable processes satisfying the following conditions:

$$
E^{n} \int_{0}^{T}\left(\xi_{n}^{j}\right)^{2}(t) d \mu_{n}^{j}(t)<\infty \quad \text { and } \quad \int_{0}^{T} \xi_{n}^{j}(t) d B_{n}^{j}(t)<\infty, \quad n \in \mathbf{Z}_{+}, 1 \leq j \leq d,
$$

almost surely with respect to the measure $P^{n}$.

Put

$$
\int_{0}^{T}\left(\xi_{n}(t), d X_{n}(t)\right):=\sum_{j=1}^{d} \int_{0}^{T} \xi_{n}^{j}(t) d X_{n}^{j}(t)
$$

Below is a simplified version of Theorem 5 of [8] fitting the case of the current paper.

Theorem 3.1. Let $\left\{X_{n}(t), \mathcal{F}_{t}^{n}, t \in[0, T], n \in \mathbf{Z}_{+}\right\}$be a sequence of semimartingales admitting representation (38) and let

(1) $\left(\xi_{n}(t), M_{n}(t), B_{n}(t), \mu_{n}(t) ; t \in \mathbf{T}\right) \stackrel{d}{\rightarrow}\left(\xi_{0}(t), M_{0}(t), B_{0}(t), \mu_{0}(t) ; t \in \mathbf{T}\right)$.

Further, for all $1 \leq j \leq d$, let

(2) $\sup _{n \geq 0} \mathrm{E}^{n} \int_{0}^{T}\left(\xi_{n}^{j}\right)^{2}(s) d \mu_{n}^{j}(s)<\infty$;

(3) $\lim _{C \rightarrow \infty} \lim \sup _{n \rightarrow \infty} \mathrm{P}^{n}\left\{\sup _{0 \leq t \leq T}\left|\xi_{n}^{j}(t)\right| \geq C\right\}=0$;

(4) $\lim _{k \rightarrow \infty} \lim \sup _{n \rightarrow \infty} \mathrm{E}^{n} \sum_{i=1}^{l_{k}} \omega_{i k} \xi_{n}^{j} \omega_{i k} B_{n}^{j}=0$;

(5) for $b>0$,

$\lim _{\delta \rightarrow 0} \limsup _{n \rightarrow \infty} \mathrm{P}^{n}\left\{\sup _{0 \leq t<t^{\prime} \leq t^{\prime \prime}<t+\delta \leq T}\left(\left|B_{n}^{j}\left(t^{\prime \prime}\right)-B_{n}^{j}\left(t^{\prime}\right)\right| \wedge\left|B_{n}^{j}\left(t^{\prime}\right)-B_{n}^{j}(t)\right|\right) \geq b\right\}=0 ;$

(6) $\lim _{k \rightarrow \infty} \lim \sup _{n \rightarrow \infty} \mathrm{E}^{n} \sum_{i=1}^{l_{k}} \omega_{i k} \xi_{n}^{j} \omega_{i k} \mu_{n}^{j}=0$;

(7) $\lim _{\delta \rightarrow 0} \lim \sup _{n \rightarrow \infty} \sup _{\sigma \in \mathcal{T}\left(\mathcal{F}^{n}\right)} \mathrm{E}^{n}\left(\mu_{n}^{j}(\sigma+\delta)-\mu_{n}^{j}(\sigma)\right)=0$.

Then the family of stochastic integrals $\int_{0}^{\cdot}\left(\xi_{n}(s), d X_{n}(s)\right)$ weakly converges, that is,

$$
\int_{0}^{\cdot}\left(\xi_{n}(s), d X_{n}(s)\right) \Rightarrow \int_{0}^{\cdot}\left(\xi_{0}(s), d X_{0}(s)\right), \quad n \rightarrow \infty .
$$

Theorem 3.2. If $\lim _{n \rightarrow \infty} m_{n}=m, \lim _{n \rightarrow \infty} \delta_{n}=\delta$, and $\lim _{n \rightarrow \infty} \sigma_{n}=\sigma>0$, then

$$
\mathrm{P}\left(A_{n}\right) \rightarrow \mathrm{P}(A), \quad n \rightarrow \infty .
$$

Proof. We apply Theorem 3.1 to prove the convergence in probability of the sets $A_{n}$. Using the notation introduced in this theorem we put

$$
\begin{gathered}
\xi_{n}^{1}(t)=\frac{m_{n}}{\delta_{n}}+\frac{1}{2} \delta_{n}, \quad M_{n}^{1}(t)=W_{t}^{n}, \quad \mu_{n}^{1}(t)=t, \quad B_{n}^{1}(t)=0 ; \\
\xi_{n}^{2}(t)=\int_{0}^{t} r_{\sigma_{n}}(t, u) d W_{u}^{n}, \quad M_{n}^{2}(t)=W_{t}^{n}, \quad \mu_{n}^{2}(t)=t, \quad B_{n}^{2}(t)=0 ; \\
\xi_{n}^{3}(t)=\left(\frac{m_{n}}{\delta_{n}}+\frac{1}{2} \delta_{n}-\int_{0}^{t} r_{\sigma_{n}}(t, u) d W_{u}^{n}\right)^{2}, \quad B_{n}^{3}(t)=t .
\end{gathered}
$$


Then we check all the assumptions of Theorem 3.1 .

Condition (1). To check that the finite-dimensional distributions of the sequence $\left\{\left(\xi_{n}^{j}(t), X_{n}^{j}(t), B_{n}^{j}(t), \mu_{n}^{j}(t)\right), 1 \leq j \leq 3\right\}$ weakly converge, one needs to prove the convergence of the corresponding characteristic functions. Moreover, the convergence can be shown only for two processes

$$
\int_{0}^{t} r_{\sigma_{n}}(t, u) d W_{u}^{n} \text { and } W_{t}^{n}
$$

The latter stochastic processes have a joint Gaussian distribution, zero expectations, and covariance function

$$
R(u, v)=\mathrm{E} W_{u}^{n} \int_{0}^{v} r_{\sigma_{n}}(v, u) d W_{u}^{n}=\int_{0}^{u \wedge v} r_{\sigma_{n}}(v, s) d s .
$$

We prove that

$$
\int_{0}^{u \wedge v} r_{\sigma_{n}}(v, s) d s \rightarrow \int_{0}^{u \wedge v} r_{\sigma}(v, s) d s, \quad n \rightarrow \infty .
$$

By Lemma 2.4, we have the pointwise convergence $r_{\sigma_{n}}(v, s) \rightarrow r_{\sigma}(v, s)$ as $n \rightarrow \infty$.

Next we find an integrable majorizing function. There exists $c>0$ such that $\sigma_{n}<\sigma+c$ for all $n$. Then inequality (11) implies that

$$
\begin{aligned}
\left|r_{\sigma_{n}}(v, s)\right|< & \sigma_{n}^{2} H(2 H-1)(v-s)^{2 H-2}+C_{T, H, \sigma_{n}} \\
\leq & (\sigma+c)^{2} H(2 H-1)(v-s)^{2 H-2} \\
& +2 H^{2}(2 H-1)^{2} \frac{T^{4 H-3}}{4 H-3}(\sigma+c)^{4} \exp \left(2 C_{T, \sigma_{n}}\right) .
\end{aligned}
$$

As before, let $\alpha=H-\frac{1}{2}>0$. Using the property of self-similarity of the kernel $r_{\sigma}$, we obtain

$$
\begin{aligned}
C_{T, \sigma_{n}} & =\int_{0}^{T} \int_{0}^{s}\left(r_{\sigma_{n}}(s, u)\right)^{2} d u d s \\
& \leq \int_{0}^{T\left(\sigma_{n} /(\sigma+c)\right)^{1 / \alpha}} \int_{0}^{s\left(\sigma_{n} /(\sigma+c)\right)^{1 / \alpha}} r_{\sigma+c}^{2}(s, u) d u d s \\
& \leq \int_{0}^{T} \int_{0}^{s} r_{\sigma+c}^{2}(s, u) d u d s<\infty .
\end{aligned}
$$

Since $2 H-2>-\frac{1}{2}$, inequality (39) provides a majorizing function. Then the Lebesgue dominated convergence theorem implies

$$
\int_{0}^{u \wedge v} r_{\sigma_{n}}(v, s) d s \rightarrow \int_{0}^{u \wedge v} r_{\sigma}(v, s) d s, \quad n \rightarrow \infty .
$$

Hence condition (1) of Theorem 3.1 is satisfied.

Condition (2). First we check condition (2) for $\left\{\xi_{n}^{2}\right\}$ :

$$
\begin{aligned}
\sup _{n \geq 0} \mathrm{E}^{n} \int_{0}^{t}\left(\int_{0}^{s} r_{\sigma_{n}}(s, u) d W_{u}^{n}\right)^{2} d s & =\sup _{n \geq 0} \int_{0}^{t} \int_{0}^{s} r_{\sigma_{n}}^{2}(s, u) d u d s \\
& \leq \sup _{n \geq 0} \int_{0}^{t\left(\frac{\sigma_{n}}{\sigma+c}\right)^{1 / \alpha}} \int_{0}^{s\left(\frac{\sigma_{n}}{\sigma+c}\right)^{1 / \alpha}} r_{\sigma+c}^{2}(s, u) d u d s \\
& \leq \int_{0}^{t} \int_{0}^{s} r_{\sigma+c}^{2}(s, u) d u d s<\infty
\end{aligned}
$$


Taking into account the preceding calculations, condition (2) for $\left\{\xi_{n}^{3}\right\}$ becomes of the form

$$
\sup _{n \geq 0} \mathrm{E}^{n} \int_{0}^{t}\left(\frac{m_{n}}{\delta_{n}}+\frac{1}{2} \delta_{n}\right)^{2} d s<\infty
$$

for $t \leq T$ and is obviously satisfied. Condition (2) for $\left\{\xi_{n}^{1}\right\}$ is also obvious, since $\mu_{n}^{3}=0$.

Condition (3). First we check this condition for $\left\{\xi_{n}^{2}\right\}$. By Corollary 2 of [․ p. 181],

$$
\mathrm{P}\left\{\sup _{t \in[0, T]} \xi_{n}^{2}(t)>c\right\} \leq 1-\Phi\left(\frac{c-4 \sqrt{2} D\left(T, \frac{\theta}{2}\right)}{\theta}\right)
$$

for all $c \geq 4 \sqrt{2} D(T, \theta / 2)$, where

$$
D(T, \theta / 2)=\int_{0}^{\theta / 2}(\ln (T / u))^{1 / 2} d u
$$

is the Dudley integral, $\theta^{2}=\sup _{t \in[0, T]} \operatorname{Var} \xi_{n}^{2}(t)$, and where $\Phi$ is the standard normal distribution function. Next we show that

$$
\sup _{t \in[0, T]} \operatorname{Var} \xi_{n}^{2}(t)<\infty
$$

Indeed,

$$
\begin{aligned}
\sup _{t \in[0, T]} \operatorname{Var} \xi_{n}^{2}(t) & =\sup _{t \in[0, T]} \operatorname{Var} \int_{0}^{t} r_{\sigma_{n}}(t, u) d W_{u}^{n} \\
& =\sup _{t \in[0, T]} \mathrm{E}\left(\int_{0}^{t} r_{\sigma_{n}}(t, u) d W_{u}^{n}\right)^{2} \\
& =\sup _{t \in[0, T]} \int_{0}^{t} r_{\sigma_{n}}^{2}(t, u) d u \\
& \leq C_{H, T} \cdot \sigma_{n}{ }^{4} \exp \left(2 \int_{0}^{t} \int_{0}^{s} r_{\sigma_{n}}^{2}(s, u) d u d s\right) \\
& \leq C_{H, T} \cdot(\sigma+c)^{4} \exp \left(2 \int_{0}^{T} \int_{0}^{s} r_{\sigma+c}^{2}(s, u) d u d s\right)=:\left(\theta^{*}\right)^{2}
\end{aligned}
$$

where

$$
C_{H, T}=2 H^{2}(2 H-1)^{2} \frac{T^{4 H-3}}{4 H-3} .
$$

In the above reasoning we used an inequality obtained in the proof of Lemma 2.2 of [7].

Now $D(T, \theta / 2) \leq D\left(T, \theta^{*} / 2\right)$ by a property of the Dudley integral, whence

$$
\mathrm{P}\left\{\sup _{t \in[0, T]}\left|\xi_{n}^{2}(t)\right|>C\right\} \leq 2\left(1-\Phi\left(\frac{C-4 \sqrt{2} D\left(T, \theta^{*} / 2\right)}{\theta^{*}}\right)\right) \rightarrow 0 \quad \text { as } C \rightarrow+\infty .
$$

Condition (3) for $\xi_{n}^{1}(t)$ obviously holds. The above inequalities show that it also holds for $\xi_{n}^{3}(t)$.

Condition (5) is obvious. 
Condition (6). It is clear that this condition holds for both $\xi_{n}^{1}$ and $\xi_{n}^{3}$. We prove that it also holds for $\xi_{n}^{2}$. Put $\Delta_{i k}:=t_{i k}-t_{i-1 k}$. Then

$$
\begin{aligned}
& \mathrm{E}^{n} \sum_{i=1}^{l_{k}} \omega_{i k} \xi_{n}^{2} \omega_{i k} \mu_{n}^{2} \\
& \quad=\mathrm{E}^{n} \sum_{i=1}^{l_{k}} \sup _{t_{i-1} \leq s<t \leq t_{i k}}\left|\int_{0}^{t} r_{\sigma_{n}}(t, u) d W_{u}^{n}-\int_{0}^{s} r_{\sigma_{n}}(s, u) d W_{u}^{n}\right| \Delta_{i k} \\
& \quad \leq 2 \mathrm{E}^{n} \sum_{i=1}^{l_{k}} \sup _{t \in\left[t_{i-1 k}, t_{i k}\right]}\left|\int_{0}^{t} r_{\sigma_{n}}(t, u) d W_{u}^{n}-\int_{0}^{t_{i-1 k}} r_{\sigma_{n}}\left(t_{i-1 k}, u\right) d W_{u}^{n}\right| \Delta_{i k} \\
& \quad \leq 2 \max _{i=1, \ldots, l_{k}} \mathrm{E}^{n} \sup _{t \in\left[t_{i-1 k}, t_{i k}\right]} \mid \int_{0}^{t} r_{\sigma_{n}}(t, u) d W_{u}^{n} \\
& \quad \leq 2 T \max _{i=1, \ldots, l_{k}}\left(\mathrm { E } ^ { n } \operatorname { s u p } _ { t \in [ t _ { i - 1 k } , t _ { i k } ] } \left(\int_{0}^{t} r_{\sigma_{n}}(t, u) d W_{u}^{n} r_{\sigma_{n}}\left(t_{i-1 k}, u\right) d W_{u}^{n} \mid \cdot T\right.\right. \\
& \left.\left.-\int_{0}^{t_{i-1 k}} r_{\sigma_{n}}\left(t_{i-1 k}, u\right) d W_{u}^{n}\right)^{2}\right)^{1 / 2}
\end{aligned}
$$

Put

$$
\Delta \xi_{n i k}^{2}(t)=\int_{0}^{t} r_{\sigma_{n}}(t, u) d W_{u}^{n}-\int_{0}^{t_{i-1 k}} r_{\sigma_{n}}\left(t_{i-1 k}, u\right) d W_{u}^{n}
$$

where $t \in\left[t_{i-1 k}, t_{i k}\right]$. Since $\inf _{t \in\left[t_{i-1 k}, t_{i k}\right]}\left\|\Delta \xi_{n i k}^{2}(t)\right\|_{U}=0$, we apply inequality (28) to the process $\Delta \xi_{n i k}^{2}(t)$ and obtain

$$
\left\|\sup _{t \in\left[t_{i-1 k}, t_{i k}\right]} \Delta \xi_{n i k}^{2}(t)\right\|_{U} \leq \frac{e \sqrt{2}}{\theta(1-\theta)} \int_{0}^{\varepsilon_{0} \theta}\left(\ln \left(\frac{\Delta_{i k}}{2 \sigma^{(-1)}(u)}+2\right)\right)^{1 / 2} d u .
$$

Lemma 2.5 implies that

$$
\sigma(h)=C h^{H-3 / 4} \quad \text { and } \quad \varepsilon_{0} \leq C \Delta_{i k}^{H-3 / 4}
$$

where $C>0$ is a certain constant.

Let $\gamma:=H-\frac{3}{4}$ and put $\theta=\frac{1}{2}$. Since $\sigma^{(-1)}(u)=(u / C)^{1 / \gamma}$, inequality (44) can be rewritten as follows:

$$
\left\|\sup _{t \in\left[t_{i-1 k}, t_{i k}\right]} \Delta \xi_{n i k}^{2}(t)\right\|_{U} \leq 4 \sqrt{2} e \int_{0}^{\frac{1}{2} C \Delta_{i k}^{\gamma}}\left(\ln \left(\frac{\Delta_{i k}}{2} \cdot\left(\frac{C}{u}\right)^{1 / \gamma}+2\right)\right)^{1 / 2} d u .
$$

The right hand side of inequality (45) approaches zero as $\Delta_{i k} \rightarrow 0$. Indeed, since

$$
\frac{\Delta_{i k}}{2} \cdot\left(\frac{C}{u}\right)^{1 / \gamma}+2>2
$$


we apply the inequality $\ln (1+x)<C_{\gamma} x^{\gamma}$ for all $x>1$, where $C_{\gamma}>0$ is a constant. As a result,

$$
\begin{gathered}
\int_{0}^{\frac{1}{2} C \Delta_{i k}^{\gamma}}\left(\ln \left(\frac{\Delta_{i k}}{2} \cdot\left(\frac{C}{u}\right)^{1 / \gamma}+2\right)\right)^{1 / 2} d u \\
\leq \int_{0}^{\frac{1}{2} C \Delta_{i k}^{\gamma}}\left(\frac{\Delta_{i k}}{2} \cdot\left(\frac{C}{u}\right)^{1 / \gamma}+1\right)^{\gamma / 2} d u .
\end{gathered}
$$

Changing the variable $z=\left(\Delta_{i k} / 2\right) \cdot(C / u)^{1 / \gamma}$, the latter integral becomes of the form

$$
\left(\frac{\Delta_{i k}}{2}\right)^{\gamma} \cdot C \gamma \cdot \int_{2^{(1-\gamma) / \gamma}}^{+\infty}(1+z)^{\gamma / 2} z^{-\gamma-1} d z .
$$

Integral (47) converges, since $\gamma / 2-\gamma-1<-1$ and $\gamma>0$. This means that the integral in (47) approaches zero as $\Delta_{i k} \rightarrow 0$ and this completes the proof of condition (6) of Theorem 3.1

Condition (4). This condition is obvious for $\xi_{n}^{1}$ and $\xi_{n}^{2}$. Thus we prove it for $\xi_{n}^{3}$. Let $a_{n}=m_{n} / \delta_{n}+\frac{1}{2} \delta_{n}$. Similarly to the proof of condition (6) we obtain

$$
\begin{aligned}
& \mathrm{E}^{n} \sum_{i=1}^{l_{k}} \omega_{i k} \xi_{n}^{3} \omega_{i k} B_{n}^{3} \\
& \quad \leq 2 T \max _{i=1, \ldots, l_{k}} \mathrm{E}^{n} \sup _{t \in\left[t_{i-1 k}, t_{i k}\right]}\left|\left(\xi_{n}^{2}(t)-a_{n}\right)^{2}-\left(\xi_{n}^{2}\left(t_{i-1 k}\right)-a_{n}\right)^{2}\right| \\
& \quad \leq 2 T \max _{i=1, \ldots, l_{k}} \mathrm{E}^{n} \sup _{t \in\left[t_{i-1 k}, t_{i k}\right]}\left|\xi_{n}^{2}(t)-\xi_{n}^{2}\left(t_{i-1 k}\right)\right| \cdot\left|\xi_{n}^{2}(t)+\xi_{n}^{2}\left(t_{i-1 k}\right)-2 a_{n}\right| \\
& \quad \leq 2 T \max _{i=1, \ldots, l_{k}}\left(\mathrm{E}^{n} \sup _{t \in\left[t_{i-1 k}, t_{i k}\right]}\left|\xi_{n}^{2}(t)-\xi_{n}^{2}\left(t_{i-1 k}\right)\right|^{2}\right. \\
& \left.\quad+\mathrm{E}^{n} \sup _{t \in\left[t_{i-1 k}, t_{i k}\right]}\left|\xi_{n}^{2}(t)-\xi_{n}^{2}\left(t_{i-1 k}\right)\right| \cdot\left|2 \xi_{n}^{2}\left(t_{i-1 k}\right)-2 a_{n}\right|\right) .
\end{aligned}
$$

Recall that we have shown that $\mathrm{E}^{n} \sup _{t \in\left[t_{i-1 k}, t_{i k}\right]}\left|\xi_{n}^{2}(t)-\xi_{n}^{2}\left(t_{i-1 k}\right)\right|^{2} \rightarrow 0$ as $\Delta_{i k} \rightarrow 0$ in the proof of condition (6).

By the Cauchy-Bunyakovskiǔ inequality,

$$
\begin{aligned}
& \mathrm{E}^{n} \sup _{t \in\left[t_{i-1 k}, t_{i k}\right]}\left|\xi_{n}^{2}(t)-\xi_{n}^{2}\left(t_{i-1 k}\right)\right| \cdot\left|2 \xi_{n}^{2}\left(t_{i-1 k}\right)-2 a_{n}\right| \\
& \quad \leq\left(\mathrm{E}^{n} \sup _{t \in\left[t_{i-1 k}, t_{i k}\right]}\left|\xi_{n}^{2}(t)-\xi_{n}^{2}\left(t_{i-1 k}\right)\right|^{2}\right)^{1 / 2} \cdot\left(\mathrm{E}^{n}\left|2 \xi_{n}^{2}\left(t_{i-1 k}\right)-2 a_{n}\right|^{2}\right)^{1 / 2} .
\end{aligned}
$$

Note that the second factor on the right hand side of inequality (49) does not depend on $t$ and thus the symbol sup is omitted. Further, the sequence

$$
\left(\mathrm{E}^{n}\left|2 \xi_{n}^{2}\left(t_{i-1 k}\right)-2 a_{n}\right|^{2}\right)^{1 / 2}
$$

is bounded. Indeed,

$$
\mathrm{E}^{n}\left|2 \xi_{n}^{2}\left(t_{i-1 k}\right)-2 a_{n}\right|^{2}=4 \int_{0}^{t_{i-1 k}} r_{\sigma_{n}}^{2}\left(t_{i-1 k}, u\right) d u+4 a_{n}^{2}
$$


Bounds (42) and Lemma 2.2 of [7] imply that the integral

$$
\int_{0}^{t_{i-1 k}} r_{\sigma_{n}}^{2}\left(t_{i-1 k}, u\right) d u
$$

is bounded and that the sequence $\left\{a_{n}\right\}$ converges. This proves that the sequence on the right hand side of the latter equality is bounded.

Hence the expression on the right hand side of inequality (49) approaches zero as $\Delta_{i k} \rightarrow 0$ and this proves condition (4) of Theorem 3.1

Condition (7) is obvious.

\section{Concluding Remarks}

The convergence in probability of the sets of maximum probability of success is proved in the problem of quantile hedging for the model of a price process involving Brownian and fractional Brownian motions. The density $d P^{*} / d P$ is expressed in terms of the Volterra kernel $r_{\sigma}(t, s)$. Some properties of $r_{\sigma}(t, s)$ are also studied.

\section{BIBLIOGRAPHY}

1. M. Bratyk and Y. Mishura, Quantile hedging with rediscounting on complete financial market, Prykladna statystyka. Aktuarna i finansova matematyka (2007), no. 2, 46-57.

2. P. Cheridito, Regularizing Fractional Brownian Motion with a View towards Stock Price Modeling, Ph.D. Thesis, Zurich, 2001, pp. 157-173. MR2715456

3. M. Hitsuda, Representation of Gaussian processes equivalent to Wiener process, Osaka J. Math. (1968), no. 5, 299-312. MR0243614(39:4935)

4. H. Föllmer and P. Leukert, Quantile hedging, Finance Stochast. (1999), no. 3, 251-273. MR 1842286 (2002g:91096)

5. V. V. Buldygin and Yu. V. Kozachenko, Metric Characterization of Random Variables and Random Processes, TViMS, Kiev, 1998; English transl., American Mathematical Society, Providence, Rhode Island, 2000. MR1743716 (2001g:60089)

6. M. A. Lifshits, Gaussian Random Functions, TViMS, Kiev, 1995; English transl., Kluwer Academic Publishers, Dordrecht, 1995. MR1472736 (98k:60059)

7. A. Melnikov and Yu. Mishura, On pricing and hedging in financial markets with long-range dependence, Math. Financ. Econ. 5 (2011), no. 1, 29-46. MR2810792(2012f:60141)

8. Yu. S. Mishura, G. M. Shevchenko, and Yu. V. Yukhnovs'kiü, Functional limit theorems for stochastic integrals with applications to risk processes and to self-financing strategies in a multidimensional market. I, Theor. Imovirnost. Matem. Statyst. 81 (2009), 114-127; English transl. in Theor. Probability and Math. Statist. 81 (2010), 131-146. MR2667315 (2011e:60069)

9. Yu. V. Kozachenko and Yu. S. Mishura, Maximal upper bounds for the moments of stochastic integrals and solutions of stochastic differential equations with respect to fractional Brownian motion with Hurst index $H<1 / 2$. I, Theor. Imovirnost. Matem. Statyst. 75 (2006), 4559; English transl. in Theor. Probability and Math. Statist. 75 (2007), 51-64. MR2321180 (2008g:60167)

Department of Mathematics, Faculty for Informatics, National University "Kyiv Mohyla ACAdemy", Skovorody Street 2, Kyiv 04070, Ukraine

E-mail address: mbratyk@ukr.net

Department of Probability Theory, Statistics, and Actuarial Mathematics, Faculty for Mechanics and Mathematics, National Taras Shevchenko University, Academician Glushkov Avenue 4E, Kiev 03127, Ukraine

E-mail address: ykoz@univ.kiev.ua

Department of Probability Theory, Statistics, and Actuarial Mathematics, Faculty for Mechanics and Mathematics, National Taras Shevchenko University, Academician Glushkov Avenue 4E, Kiev 03127, Ukraine

E-mail address: myus@univ.kiev.ua 